\title{
NEW PERSPECTIVES IN TURBULENCE: SCALING LAWS, ASYMPTOTICS, AND INTERMITTENCY*
}

\author{
G. I. BARENBLATT ${ }^{\dagger}$ AND A. J. CHORIN ${ }^{\dagger}$
}

\begin{abstract}
Intermittency, a basic property of fully developed turbulent flow, decreases with growing viscosity; therefore classical relationships obtained in the limit of vanishing viscosity must be corrected when the Reynolds number is finite but large. These corrections are the main subject of the present paper. They lead to a new scaling law for wall-bounded turbulence, which is of key importance in engineering, and to a reinterpretation of the Kolmogorov-Obukhov scaling for the local structure of turbulence, which has been of paramount interest in both theory and applications. The background of these results is reviewed, in similarity methods, in the statistical theory of vortex motion, and in intermediate asymptotics, and relevant experimental data are summarized.
\end{abstract}

Key words. turbulence, intermittency, scaling, wall-bounded turbulence, local structure, statistical theory

AMS subject classifications. $76 \mathrm{~F} 10,76 \mathrm{~F} 05,76 \mathrm{C} 05$

PII. S0036144597320047

1. Introduction. Self-similar states and the corresponding scaling laws are the cornerstones of statistical theories in physics; in the case of turbulence, the best known self-similar states are found in the intermediate region in wall-bounded turbulence, whose mean structure has been widely thought to be well described by the von Kármán-Prandtl universal logarithmic law of the wall, and in the intermediate region of local structure, for which Kolmogorov and Obukhov proposed their well-known scaling laws. These laws have been put to use in a wide variety of applications and constitute a substantial fraction of the accepted wisdom in turbulence theory. The goal of the present paper is to reexamine much of that accepted wisdom.

Our basic premise is that turbulence can be described by the Navier-Stokes equations. As the viscosity tends to zero, these equations formally converge to the Euler equations, but their solutions acquire temporal and spatial fluctuations with a limiting behavior that is at this time imperfectly understood. However, certain average properties of the flow, which will be identified as we proceed, have well-defined limits as the viscosity tends to zero, and the existence of these limits is the basis for expansions in a small parameter that tends to zero as the viscosity tends to zero.

In the case of wall-bounded turbulence, our argument will show that the classical von Kármán-Prandtl law should be abandoned and replaced, when the viscosity is small but finite, by a scaling (power) law. The well-known graphs that seem to exhibit the classical von Kármán-Prandtl law are misleading for reasons we shall explain. In fact, arguments commonly used in favor of the von Kármán-Prandtl law support our conclusions once they are properly understood.

In the case of local structure, the classical Kolmogorov-Obukhov scaling of the second- and third-order structure functions is exact in the limit of vanishing viscosity, when the turbulence is most intermittent and least organized. When the viscosity

${ }^{*}$ Received by the editors April 16, 1997; accepted for publication (in revised form) September 19, 1997. This research was supported in part by the Applied Mathematical Sciences subprogram of the Office of Energy Research, U.S. Department of Energy, under contract DE-AC03-76-SF00098, and in part by NSF grants DMS94-14631 and DMS89-19074.

http://www.siam.org/journals/sirev/40-2/32004.html

$\dagger$ Department of Mathematics and Lawrence Berkeley National Laboratory, University of California, Berkeley, CA 94720 (chorin@math.berkeley.edu). 
is nonzero (the Reynolds number is large but finite), Reynolds-number-dependent corrections to the Kolmogorov-Obukhov scaling of these structure functions appear and are due to a viscosity-induced reduction in intermittency. These conclusions are antithetical to common assumptions according to which the Kolmogorov-Obukhov scaling somehow omits the effects of the spottiness or intermittency that is inherent in turbulence and must be corrected accordingly. For higher order structure functions the vanishing viscosity limit ceases to exist because of intermittency, and thus the Kolmogorov-Obukhov scaling fails for these structure functions not because it must be corrected for intermittency, but because of the intermittency that it already describes.

It is worth noting that though both the Kolmogorov-Obukhov scaling and the von Kármán-Prandtl law are widely used and accepted, they have not been immune from all criticism. In the case of local structure, Landau (see Landau and Lifshitz [36]) made a case for "intermittency corrections" shortly after the original laws were formulated, and the need for such corrections was endorsed by Kolmogorov and Obukhov themselves [35], [42]. An elaborate statistical theory of turbulence has arisen in recent decades, and while it has not been particularly successful elsewhere, it is widely believed to offer a good and sufficient explanation not only of the Kolmogorov-Obukhov scaling but also of the need for correcting it (see, e.g., [26], [37], [38]). Laws other than the von Kármán-Prandtl law have been long known to fit the data near a wall (see [46]), and a self-consistent alternative has been offered by one of the present authors starting with the monograph [1].

In both problems the focus is on an intermediate range of scales, between the scales ruled by outside forcing and the scales where viscosity is important; this will bring us into the realm of intermediate asymptotics. In addition to intermediate asymptotics, our mathematical tools include similarity methods as well as vanishingviscosity asymptotics motivated by the statistical mechanics of vortex motion, all of which will be summarized in the next two sections. In the sections that follow we shall consider the wall region and local structure, with special attention to the "overlap" asymptotic argument and to the experimental data. We shall then turn to a unified treatment of intermittency and offer conclusions.

We wish to express our belief that the combination of similarity methods with asymptotics based on a statistical theory constitutes a step forward in the analysis of turbulence from first principles, and that it has the signal advantage of placing turbulence theory within a broad framework shared by other nonequilibrium theories in statistical physics.

The example we shall dwell on most is fully developed turbulent flow in a pipe, and in order to motivate the theoretical discussion in the next few sections we begin by summarizing its salient features.

Consider a long cylindrical pipe with a circular cross-section and the average flow in its working section, i.e., far from its inlet and outlet. Data about turbulent flow are generally presented in a dimensionless form, making possible a unified description of flows of different fluids, in pipes of various diameters, etc. This dimensionless description should be independent of the choice of the magnitude of the basic units of measurement. In particular, it is customary to represent $u$, the average longitudinal velocity in a pipe, as

$$
\phi=u / u_{*},
$$

where $u_{*}$ is the "dynamic" or "friction" velocity that defines the appropriate velocity 


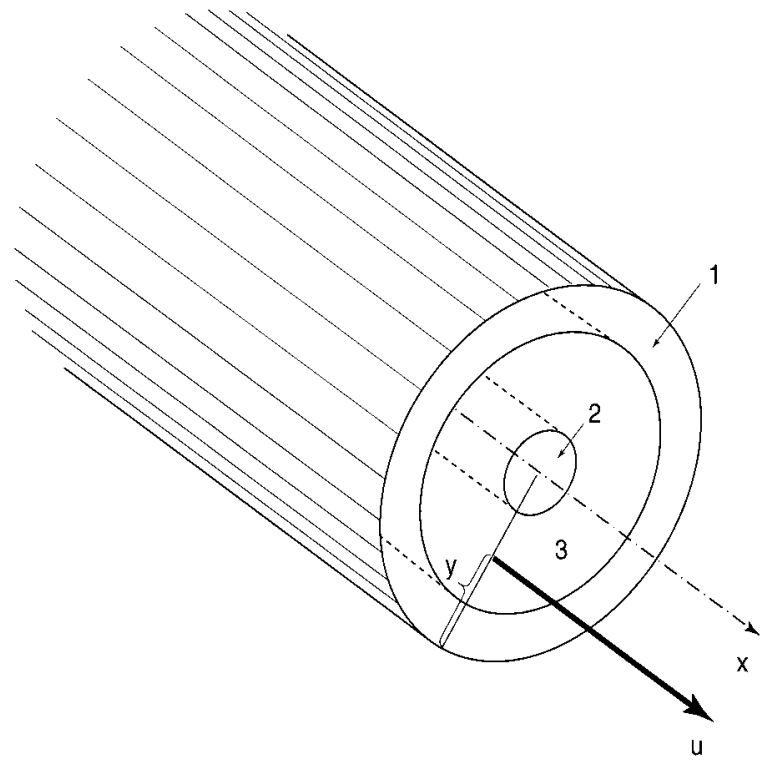

FIG. 1. Schematic view of flow in a pipe. 1. Viscous sublayer. 2. Near-axis region. 3. Intermediate region.

scale:

$$
u_{*}=\sqrt{\tau / \rho},
$$

where $\rho$ is the density of the fluid and $\tau$ is the shear stress at the pipe's wall defined as

$$
\tau=\frac{\Delta p}{L} \frac{d}{4}
$$

Here $\Delta p$ is the pressure drop over the working section of the pipe, $L$ is the length of the working section, and $d$ is the pipe's diameter. The dimensionless distance from the pipe wall is represented as

$$
\eta=\frac{u_{*} y}{\nu}
$$

where $y$ is the actual distance from the wall and $\nu$ is the kinematic viscosity. Note that the length scale $\nu / u_{*}$ implicit in (1.4) is typically very small - of the order of tens of microns or even less in some of the data discussed below.

An important parameter in the problem is the Reynolds number

$$
R e=\frac{\bar{u} d}{\nu},
$$

where $\bar{u}$ is the mean velocity averaged over the cross-section, i.e., the average fluid flux divided by the area of the cross-section. When the Reynolds number Re is large, one observes that the cross-section is divided into three parts (Figure 1): a thin ring (1) near the wall, where the velocity gradient is so large that the shear stress due to molecular viscosity, i.e., to the rate of momentum transfer by the thermal motion of 
the fluid's molecules, is comparable to the turbulent shear stress, i.e., to the rate of momentum transfer due to the turbulent vortices. This is the viscous sublayer. In a cylinder (2) surrounding the pipe's axis the velocity gradient is small and the average velocity is close to its maximum. We shall focus on the intermediate region (3) which occupies most of the cross-section.

During the last sixty years two contrasting laws for the velocity distribution in the intermediate region could be found in the literature (see, e.g., Schlichting [46]): the first is the "scaling" or "power" law,

$$
\phi=C \eta^{\alpha},
$$

where the $C$ and $\alpha$ are parameters independent of $\eta$ but believed to depend weakly on $R e$. Laws such as (1.6) were used by engineers in the early years of turbulence research. The second law found in the literature is the "universal," Reynolds number independent logarithmic law,

$$
\phi=\frac{1}{\kappa} \ln \eta+B,
$$

where $\kappa$ (von Kármán's constant) and $B$ are assumed to be "universal," i.e., Reindependent, constants. The values of $\kappa$ in the literature range between .36 and .44, and the values of $B$ range between 5 and 6.3 .

A widely accepted derivation of the universal logarithmic law (1.7), due originally to von Kármán [30] and Prandtl [44], who used some additional assumptions, and in its final form to Landau and Lifshitz [36], proceeds as follows. Assume that the velocity gradient $\partial_{y} u\left(\partial_{y} \equiv \frac{\partial}{\partial y}\right)$ in the intermediate region (2) of Figure 1 depends on the following variables: the coordinate $y$, the shear stress at the wall $\tau$, the pipe diameter $d$, and the properties of the fluid: its kinematic viscosity $\nu$ and density $\rho$. We consider the velocity gradient $\partial_{y} u$ rather than $u$ itself because the values of $u$ depend on the flow in the viscous sublayer where the assumptions we shall use are not valid. Thus

$$
\partial_{y} u=f(y, \tau, d, \nu, \rho) .
$$

Dimensional analysis (see section 3 below) gives

$$
\partial_{y} u=\frac{u_{*}}{y} \Phi(\eta, R e), \quad R e=\frac{\bar{u} d}{\nu}, \quad \eta=\frac{u_{*} y}{\nu},
$$

where $\Phi$ is a dimensionless function. The same kind of dimensional analysis gives for the "friction" velocity

$$
\frac{u_{*} d}{\nu}=\frac{\bar{u} d}{\nu} \cdot F(R e),
$$

where $F$ is a dimensionless function and the Reynolds number $R e$ is given by equation (1.5). Thus equation (1.8) can be rewritten in the form

$$
\partial_{\eta} \phi=\frac{1}{\eta} \Phi(\eta, R e), \quad \phi=\frac{u}{u_{*}} .
$$

Outside the viscous sublayer, $\eta$ is large - of the order of several tens and more; in the kind of turbulent flow we consider the Reynolds number $R e$ is also large, of the order 


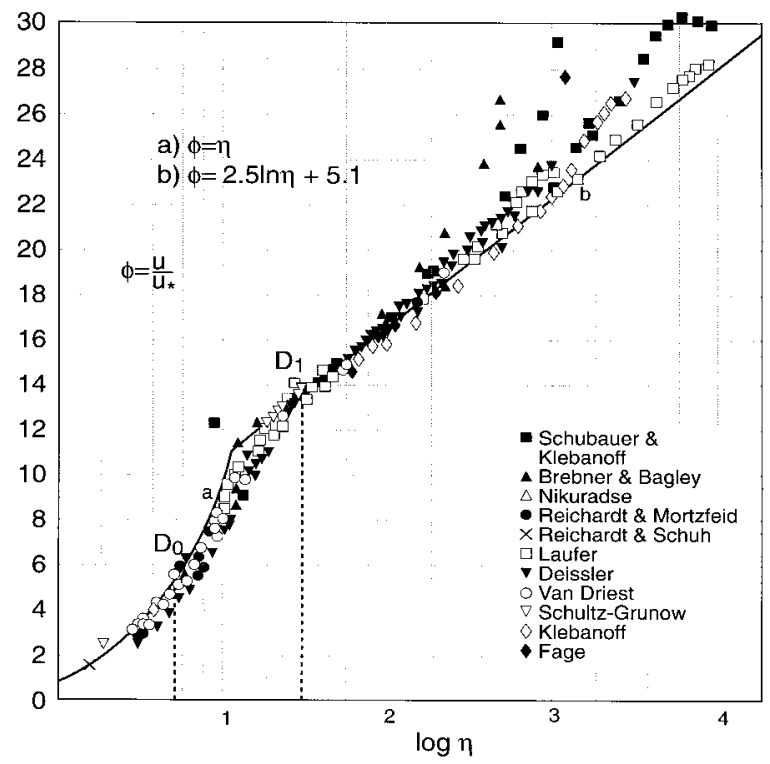

FIG. 2. The comparison of the universal logarithmic law with experiment (after Monin and Yaglom [39]).

of $10^{4}$ at least. It was therefore natural to assume that for such large values of $\eta$ and $R e$ the function $\Phi$ no longer varied with its argument and could be replaced by its limiting value $\Phi(\infty, \infty)=\kappa^{-1}$. Substitution into (1.11) yielded

$$
\partial_{\eta} \phi=\frac{1}{\kappa \eta},
$$

and an integration yielded the logarithmic law (1.7).

However, as we shall see in detail below, there is no overwhelming reason to assume that the function $\Phi$ has a constant, nonzero limit as its arguments tend to infinity, nor that the integration constant remains bounded as Re tends to infinity. When either assumption fails other conclusions must be reached.

It is often stated that the universal logarithmic law (1.7) is in satisfactory agreement with the experimental data both in pipes and in boundary layers. Graphs such as those in Figure 2 (drawn after Monin and Yaglom [39]) are adduced as evidence. However, the scaling law (1.6) has also found experimental support, provided the dependence of the quantities $\alpha$ and $C$ on the Reynolds number was properly taken into account. Indeed, Schlichting [46], following Nikuradze [41], showed that the experimental data agree with the scaling law over practically the whole cross-section of a pipe. We shall show that if one plots experimental points on a graph without regard to Reynolds number, as was done in the preparation of Figure 2, then it is natural but in fact misleading to focus on the envelope of the family of Reynolds-number-dependent curves, which happens to be close to the graph of the von Kármán-Prandtl law.

In later sections, after appropriate preliminaries, we shall discuss which of these laws, if any, best describes turbulent flow of fluids such as air or water. This question is of great practical as well as theoretical significance. We shall then use our conclusions in the discussion of local structure, and more generally, in a discussion of scaling and intermittency in turbulence. 
2. The near-equilibrium statistical theory of turbulence. It is well known that if one adds to a hyperbolic system of equations (for example, to the equations of inviscid gas dynamics) a suitable viscous term with a small viscosity coefficient and then makes the viscosity tend to zero, one obtains in the limit a suitable solution of the equations with zero viscosity. On the other hand, if one adds to the hyperbolic system a dispersive term (for example, a small coefficient multiplying a third derivative) and then decreases the small coefficient, one observes in general rapid oscillations in the solution which do not disappear as the limit is approached. Due to the interaction and self-interaction of vortices, the three-dimensional Navier-Stokes equations partake of both hyperbolic and dispersive properties. For example, the motion of vortex lines can be described, in certain approximations, by equations of Schroedinger type [32], which is one of the most common examples of a dispersive equation. As a result, one cannot in general expect individual solutions of the Navier-Stokes equations to be well behaved in the limit of vanishing viscosity [6]. However, it turns out that one can expect certain average properties of collections of solutions to be well behaved as the viscosity decreases, a fact that will turn out to be important below. To explain why this is so one has to make a short detour through the statistical theory of turbulence. The goal of this statistical theory is to understand and quantify the behavior of ensembles of solutions of the Navier-Stokes equations; experience in fluid mechanics as well as in other parts of physics suggests that such ensembles are much more amenable to analysis than the individual solutions.

It is natural to focus first on stationary random solutions of the Euler or NavierStokes equations, just as it is natural in the kinetic theory of gases to focus first on stationary distributions of the momenta and positions of particles. A stationary random solution in turbulence is the natural generalization of a statistically steady state in a system of $N$ particles; it is an ensemble (i.e., a collection) of solutions, each one of whose members may be varying in time, even rapidly. But the ensemble has the property that its averages and other statistical properties are invariant in time. This is analogous to the "equilibrium" solutions in the kinetic theory of gases, which describe systems in which each molecule is moving and undergoing collisions, but the average properties of the collection of particles are stationary.

Stationary random solutions are important because they may attract others; i.e., if one starts with a nonstationary random solution, in which even the averages are time varying, one may expect that after some time interval the averages will become stationary, and in particular one may be able to replace long-time averages of individual members of the ensemble by averages over a stationary statistical solution (i.e., over the appropriate ensemble of solutions with its time-independent statistics). In addition, nonstationary statistical solutions depend on their initial conditions and few general conclusions can be reached about them. When time averages can be replaced by averages over a stationary statistical solution, the latter is called ergodic. It is understood that stationary solutions may provide only a partial description of real solutions; in turbulence, this partial description often applies to the small scales, but not exclusively so; for example, the large-scale flow outside the viscous sublayer in the center section of a long pipe can be viewed as stationary, in the sense that its averages are time-invariant, and can in principle be related to averages over an ensemble of solutions that satisfy the same boundary conditions. The explanation of how a nonrandom equation can produce a random solution belongs to the realm of chaos theory [19]. 
Statistically stationary flows come in two flavors: equilibrium and nonequilibrium. An equilibrium is what one finds after a long time in an isolated system or a portion of an isolated system. One can generally assume that at equilibrium, every individual solution (member of the ensemble) that satisfies the boundary conditions has a probability $P$ of being observed in an experiment, with $P=\frac{1}{Z} e^{-\beta H}$, where $H$ is the kinetic energy of the solution, $\beta$ is an "inverse temperature," and $Z$ is a normalizing factor which ensures that the sum of all probabilities is one. In turbulence, the "inverse temperature" $\beta$ is not necessarily related to what one usually thinks of as the temperature of the fluid but is a more abstract concept, related to the energy of the turbulence. This formula for $P$ is known as Gibbs's formula, and an ensemble in which this formula holds is known as a Gibbsian ensemble. In a Gibbsian ensemble, there is no loss of energy nor any transport of mass or momentum from one point to another or to a wall. A full discussion of Gibbsian ensembles in fluid mechanics can be found in [18], [19], [20], [21].

Nonequilibrium steady states are the analogs of what one obtains in kinetic theory when one considers, for example, the distribution after a long time of velocities and momenta of gas particles between two walls at different temperatures. That distribution of momenta and locations is stationary but not Gibbsian. Unlike a Gibbsian equilibrium, it allows for the irreversible transport of mass, momentum, and energy across the system.

The great discovery of Onsager, Callen, and Welton (see [15]) is that in a system not too far from a Gibbsian equilibrium, nonequilibrium properties (e.g., transport coefficients) can be evaluated on the basis of equilibrium properties. An example is heat capacity, which is perfectly well defined at equilibrium, but measures the response of a system to external perturbations. Most of the theory of nonequilibrium processes deals with systems not far from equilibrium; its machinery, for example, the formalism for calculating properties such as energy loss, is not applicable except near equilibrium. Clearly, turbulence is not in Gibbsian equilibrium, in particular because it features an irreversible energy transfer from large to small scales or of momentum from the interior to the walls. The interesting question is: can turbulence be viewed as a small perturbation of a suitable Gibbsian equilibrium? The key word here is "suitable" and the answer is positive; this positive answer greatly simplifies the analysis of statistical solutions of the Euler and Navier-Stokes equations. An estimate of the time available for the small scales to settle to equilibrium, compared with the time scale of overall decay, will be given in section 6 below.

Statistical equilibria in vortex systems and their limiting behavior have been studied by a variety of numerical and analytical methods, some of which exploit an analogy with the vortex-dominated phase transitions that occur in superfluid and superconducting systems. A major conclusion of the available analyses is that the postulated equilibria exist and exhibit velocity correlation and structure functions up to order 3 that are consistent with the Kolmogorov scaling discussed below. Typical flows have a vorticity that is highly concentrated in small volumes, and are thus highly intermittent. A consequence of this analysis is that one can expect correlation and structure functions of low order for Navier-Stokes flows to have a well-behaved limit as the viscosity tends to zero. As noted above, it is not claimed that individual solutions of the Navier-Stokes equations converge to an Euler limit (and indeed, in the case of wall-bounded flow, this is clearly false [31]). Further, the precise nature of the convergence of the random solutions as the viscosity tends to zero remains open; 
all that is asserted is that certain moments up to order 3 have limits as the viscosity tends to zero.

3. Intermediate asymptotics, scaling laws, and similarity. Fluid dynamicists are familiar with the concept of dynamic Reynolds number similarity: if one has found a flow in a given geometry, with a length scale $L$, viscosity $\nu$, and velocity scale $U$, one can find a flow in a similar geometry, with a different length scale and a different viscosity, by scaling the velocity so that the Reynolds number $R e=U L / \nu$ is the same; in other words, if the length scale and the viscosity change, one can obtain a solution of the new problem by multiplying ("scaling") the velocity field by the appropriate constant that keeps Re fixed. We wish to generalize this simple analysis of the effects of changes in scales.

We first note that all of the problems we shall discuss involve a range of scales intermediate between very large and very small scales, and our analyses will be valid only in that range. We shall thus be performing intermediate asymptotics; a function $u=u(s)$ of the independent variable $s$ has an intermediate asymptotic expansion if that expansion is asymptotic in a range $S_{1}<<s<<S_{2}$ but not beyond. A simple example of intermediate asymptotics is afforded by the asymptotic solution $T$ of the heat equation with heat conductivity $\kappa$ :

$$
T=\frac{Q}{2 \sqrt{\pi \kappa t}} e^{-x^{2} / 4 \kappa t},
$$

where $x$ is the space variable, $t$ is the time, and $Q$ is a constant determined by the initial data, assumed to have compact support. Note that this is the temperature distribution due to a point source of strength $Q$ at the origin; it provides a description of the temperature field on scales at which the support of the data can be viewed as small, i.e., on long enough scales after enough time. On the other hand, every heat flow problem refers to a finite rather than an infinite slab, and thus the solution (3.1) breaks down on scales and is at times large enough for finite-size effects to be important. This solution is thus asymptotic to the full solution of the heat equation when

$$
\frac{h^{2}}{\kappa} \ll t \ll \frac{\lambda^{2}}{\kappa},
$$

where $\lambda$ is the length of the slab in which the solution is sought and $h$ is the size of the support of the initial data. This solution has a property of self-similarity: temperature distributions at various values of $t$ can be found from each other by similarity transformations, which we now define.

Consider a physically meaningful relation between physical variables:

$$
y=f\left(x_{1}, x_{2}, \ldots, x_{k}, c\right),
$$

where the arguments $x_{1}, x_{2}, \ldots$ have independent dimensions while the dimensions of $y$ and $c$ are monomials in the powers of the dimensions of the $x_{i}$ :

$$
\begin{aligned}
& {[y]=\left[x_{1}\right]^{p} \ldots\left[x_{k}\right]^{r},} \\
& {[c]=\left[x_{1}\right]^{q} \ldots\left[x_{k}\right]^{s} .}
\end{aligned}
$$

Here $[x]$ denotes the dimensions of the quantity $x$, and for simplicity we restrict ourselves to the case of a single argument $c$ with dependent dimensions. 
A physical relationship similar to (3.3) must hold for all observers even if they use a different system of physically equivalent units having different magnitudes. The change from one observer to another is expressed by the transformation of the values of $y, x_{1}, \ldots, x_{k}, c$, of the form

$$
x_{1}^{\prime}=A_{1} x_{1}, \ldots, \quad x_{k}^{\prime}=A_{k} x_{k}, \quad y^{\prime}=A_{1}^{p} \ldots A_{k}^{r} y, \quad c^{\prime}=A_{1}^{q} \ldots A_{k}^{s} c .
$$

Such transformations form a group; the invariants of the group, i.e., the quantities which remain invariant after the transition from one observer to the next, are obviously

$$
\Pi=\frac{y}{x_{1}^{p} \ldots x_{k}^{r}}, \quad \Pi_{1}=\frac{c}{x_{1}^{q} \ldots x_{k}^{s}} ;
$$

thus the invariant form of equation (3.3) is

$$
\Pi=\Phi\left(\Pi_{1}\right),
$$

where $\Phi$ is a dimensionless function. A comparison of equations (3.3) and (3.6) shows that the function $f\left(x_{1}, \ldots, x_{k}, c\right)$ has the generalized homogeneity property

$$
f\left(x_{1}, \ldots, x_{k}, c\right)=x_{1}^{p} \ldots x_{k}^{r} \Phi\left(\frac{c}{x_{1}^{q} \ldots x_{k}^{s}}\right) .
$$

These considerations belong to standard dimensional analysis.

Consider now what happens when the variable $\Pi_{1}$ is small, $\Pi_{1}<<1$. In such cases one is accustomed to assume that the function $\Phi$ can be replaced by the constant $C=\Phi(0)$. If this is indeed true, the problem is greatly simplified; for small enough $\Pi_{1}$ one can replace equation (3.1) by the simpler relation

$$
y=C x_{1}^{p} \ldots x_{k}^{r} .
$$

Here $C$ is a single constant to be determined, and the parameter $c$ completely disappears from the equation for small $\Pi_{1}$. The powers $p, \ldots, r$ can be found by simple dimensional analysis. When this situation holds, one says that one has complete similarity in the parameter $\Pi_{1}$. Complete similarity in the parameter $1 /$ Re, where Re is the Reynolds number, is known as Reynolds number similarity. The strong implicit assumption here is that as $\Pi_{1} \rightarrow 0, \Phi$ tends to a constant nonzero limit $C$. This is what was assumed in the derivation of the von Kármán-Prandtl logarithmic law in the introduction. However, it is obvious that in general complete similarity does not hold; in general, there is no reason to believe that $\Phi$ has a finite nonzero limit when $\Pi_{1} \rightarrow 0$, and the parameter $\Pi_{1}$, far from disappearing, may well become essential, even when, or particularly when, it is small.

Here, however, there is an important solvable special case. Assume that $\Phi$ has no nonzero finite limit when $\Pi_{1}$ tends to zero, but that in the neighborhood of $\Pi_{1}=0$ one has for $\Phi$ a representation of the form

$$
\Phi\left(\Pi_{1}\right)=C \Pi_{1}^{\alpha}+\ldots
$$

for some $C$ and $\alpha$, where the dots represent smaller terms. Substituting (3.9) into (3.6) for $\Pi_{1}$ small we find

$$
\Pi=C \Pi_{1}^{\alpha},
$$


or, returning to dimensional variables,

$$
y=C x_{1}^{p-\alpha q} \ldots x_{k}^{r-\alpha s} c^{\alpha} ;
$$

i.e., the power relation is of the same general form as in (3.8), but with two essential differences: the powers of the variables $x_{i}, i=1, \ldots, k$, cannot be obtained by dimensional analysis, because $\alpha$ is unknown, and must be derived by an additional, separate analysis, and the argument $c$ has not disappeared from the resulting relation. We refer to such cases as cases of incomplete similarity in the parameter $\Pi_{1}$ : a scaling law is obtained, however the parameter $c$ does not disappear but enters that law, albeit only in a certain well-defined power combination with the parameters $x_{1}, \ldots, x_{k}$. Although the determination of the parameter $\alpha$ requires an effort beyond dimensional analysis, the relation (3.11) has a "scaling" (power) form. Such scaling relations have a long history in engineering, where a widely shared opinion held, until recently, that since they cannot be obtained from dimensional considerations, they were nothing more than empirical correlations. In fact they are merely a more complicated case of similarity.

Note that the relation (3.9) makes sense only for $\Pi_{1} \neq 0$, while the neglect of the lower order terms in that equation is legitimate only when $\Pi_{1}$ is not too large. Thus conclusions based on equation (3.10) also constitute intermediate asymptotics, as defined above.

As an application of these ideas, consider a problem which has a qualitative connection with the fluid mechanics of wall-bounded turbulent flows, as will appear in what follows. Consider the equation

$$
u^{\prime}=\frac{1}{\ln (1 / \delta)} \frac{u}{y},
$$

where the prime denotes differentiation with respect to $y, y>0, u$ is subject to the boundary condition $u(\delta)=1$, and $\delta$ is a small parameter; we are interested in what happens when $\delta$ is small. One can view $\delta$ as a dimensionless viscosity, and thus $\delta^{-1}$ is analogous to a Reynolds number.

An obvious piece of erroneous reasoning proceeds as follows. For $\delta$ small, $u^{\prime}$ is approximately zero, and thus $u$ is a constant, which can only be the constant 1 . We can derive the same false result for small $y$ and $\delta$ by an assumption of complete similarity: equation (3.12) is homogeneous in the dimensions of $u$ and $y$, and thus one can view both of these variables as dimensionless. By construction, $\delta$ must be dimensionless. The scaling relation between these variables then takes the form

$$
u=\Phi(\delta, y),
$$

and if one assumes that for $\delta, y$ small $\Phi$ is constant, one finds again that $u$ is a constant that can only be the constant 1 .

However, both of these arguments are in error. Equation (3.12) has the following solution that satisfies the boundary condition:

$$
u(y)=\left(\frac{y}{\delta}\right)^{\frac{1}{\ln (1 / \delta)}} .
$$

Note that for any positive value of $\delta$ this solution constitutes a power law and is not a constant. We can obtain this solution for small $y$ and $\delta$ by assuming that the problem has incomplete similarity in the variable $y$ and no similarity in the variable $\delta$; this leads to a solution of the form $u=A(\delta) y^{\alpha(\delta)}$. A substitution into equation (3.12) yields the unknown functions $A(\delta), \alpha(\delta)$ and the correct solution. 


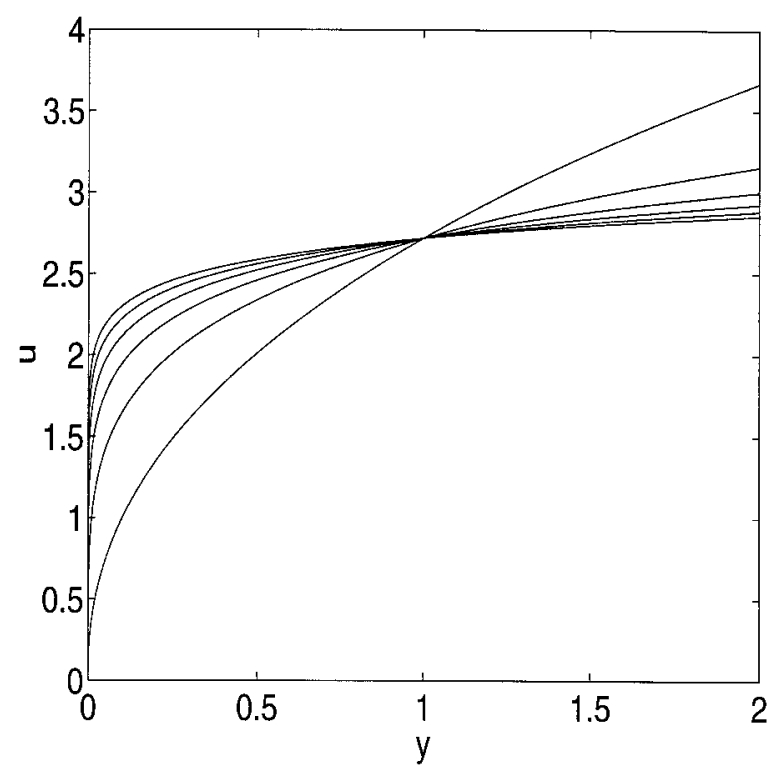

FIG. 3. The solutions of the model equation (3.12) for $\delta=10^{-n}, n=1, \ldots, 6$.

An important remark can now be made. Consider the solution (3.14) and, for a nonzero value of $y$, consider its limit as $\delta \rightarrow 0$. One can easily see that

$$
\left(\frac{y}{\delta}\right)^{\frac{1}{\ln (1 / \delta)}}=\exp \left(\frac{\ln (y / \delta)}{\ln (1 / \delta)}\right)=\exp \left(\frac{\ln (1 / \delta)+\ln (y)}{\ln (1 / \delta)}\right),
$$

and thus, as $\delta \rightarrow 0, u \rightarrow e$; i.e., the limit of (3.12) for $y>0$ is the constant $e$. As we have found from the (false) assumption of complete similarity, the limit of $u$ is a constant, but it is not the same constant as was obtained from the assumption of complete similarity. Furthermore, for a finite value of $\delta$, however small, $u$ cannot be viewed as constant everywhere; for $y<\delta, u$ is not equal to $e$, and for $y$ large enough, $u$ cannot be approximated by $e$ either. The approximate equality $u \sim e$ holds, for small but finite $\delta$, only in an intermediate range where $y=O(1)$, and it constitutes another example of intermediate asymptotics. The solutions of equation (3.12) are plotted in Figure 3 for various values of $\delta$. For a general discussion of similarity and intermediate asymptotics, see [1].

These remarks about equation (3.12) will find a counterpart in the discussion of the intermediate layer in wall-bounded turbulence as well as in the problem of local structure.

4. The intermediate region in wall-bounded turbulence. We now turn to a discussion of the intermediate region in wall-bounded turbulence. For the sake of definiteness we shall discuss mostly flow in a pipe; analogous considerations apply to turbulent boundary layers, but in this case there are additional factors that will be considered separately elsewhere.

We already derived in the introduction the general relation

$$
\partial_{y} u=\frac{u_{*}}{y} \Phi(\eta, R e), \quad R e=\frac{\bar{u} d}{\nu}, \quad \eta=\frac{u_{*} y}{\nu},
$$

where $\Phi$ is a dimensionless function. An equivalent form of this equation was found 
to be

$$
\partial_{\eta} \phi=\frac{1}{\eta} \Phi(\eta, R e), \quad \phi=\frac{u}{u_{*}} .
$$

By making the assumption of complete similarity, i.e., $\Phi$ is constant for sufficiently large values of its argument, $\Phi(\infty, \infty)=\kappa^{-1}$, one obtains after an integration, and provided the integration constant is finite, the von Kármán-Prandtl logarithmic law of the wall (1.7). Of course, our knowledge of the Navier-Stokes equations and of their solutions is not sufficient to decide whether such a limit exists. Assume, as suggested in the previous section, that this limit does not exist, but that at large $\eta$ the function $\Phi$ can be represented as a power of the form

$$
\Phi(\eta, R e)=A \eta^{\alpha},
$$

where the quantities $A$ and $\alpha$ may depend on the Reynolds number. As in the example at the end of the preceding section, we are assuming incomplete similarity in the parameter $\eta$ but no similarity nor any other invariance in the parameter $R e$. Hence,

$$
\partial_{\eta} \phi=A \eta^{\alpha-1} .
$$

By integration, equation (4.4) yields

$$
\phi=\frac{A}{\alpha} \eta^{\alpha}+\text { constant. }
$$

The scaling law (1.6) is obtained if one sets $C=A / \alpha$ and sets the additive constant equal to zero. This last condition is an independent statement; it is not a consequence of the no-slip condition at the wall because equation (4.5) is not valid in the viscous sublayer near the wall. The justification for the dropping of the additive constant is the comparison with experiment. Making explicit the dependence of $A$ and $\alpha$ on Re, we obtain

$$
\Phi(\eta, R e)=A(R e) \eta^{\alpha(R e)},
$$

in conjunction with the general relations

$$
\partial_{y} u=\frac{u_{*}}{y} \Phi(\eta, R e) \quad \text { or } \quad \partial_{\eta} \phi=\frac{1}{\eta} \Phi(\eta, R e) .
$$

An important conclusion has been reached: the power law (1.6) and the logarithmic law (1.7) can be derived with equal rigor but from different assumptions. The universal logarithmic law is obtained from the assumption of complete similarity in both $\eta$ and Re; physically, this assumption means that neither the molecular viscosity $\nu$ nor the pipe diameter $d$ influences the flow in the intermediate region. The scaling law (1.6) is obtained from an assumption of incomplete similarity in $\eta$ and no similarity in $R e$; this assumption means that the effects of both $\nu$ and $d$ are perceptible in the intermediate region.

Note immediately a clear-cut difference between the cases of complete and incomplete similarity. In the first case the experimental data should cluster in the traditional $(\ln \eta, \phi)$ plane $\left(\phi=u / u_{*}, \eta=u_{*} y / \nu\right)$ on the single straight line of the logarithmic law. In the second case the experimental points occupy an area in the $(\ln \eta, \phi)$ plane. 
Both similarity assumptions are very specific. The possibility that $\Phi$ has no nonzero limit yet cannot be represented asymptotically, as a power of $\eta$ has not been excluded. Both assumptions must be subjected to careful scrutiny. In the absence of reliable, high-Re numerical solutions of the Navier-Stokes equation and of an appropriate rigorous theory, this scrutiny must be based on careful comparison with experimental data.

We now specify the conditions under which we may expect (4.6) to hold, and narrow down the possible choices for $A(R e)$ and $\alpha(R e)$ (see [2], [3], [4], [5], [6]). We expect equation (4.6) to hold in fully developed turbulence. Experiment shows that it is not possible to view fully developed turbulence as a single, well-defined state with properties independent of $R e$. We may expect a single, well-defined, fully turbulent regime in the limit of infinite Reynolds number, but experiment, even in the largest facilities, shows that what anyone would consider fully developed turbulence still exhibits a perceptible dependence on Re. We thus define fully developed turbulence as turbulence whose mean properties (for example, the parameters $A$ and $\alpha$ in (4.6)) vary with Reynolds number like $K_{0}+K_{1} \varepsilon$, where $K_{0}, K_{1}$ are constants and $\varepsilon$ is a small parameter that tends to zero as Re tends to infinity, and is small enough so that its higher powers are negligible, yet not so small that its effects are imperceptible in situations of practical interest; the latter condition rules out choices such as $\varepsilon=$ $(R e)^{-1}$. Under these conditions we expect $A(R e)$ and $\alpha(R e)$ in (4.6) to have the form

$$
A(R e)=A_{0}+A_{1} \varepsilon, \quad \alpha(R e)=\alpha_{0}+\alpha_{1} \varepsilon,
$$

where $A_{0}, A_{1}, \alpha_{0}, \alpha_{1}$ are universal constants. Here we have implicitly used a principle that can be derived from the statistical theory of section 2, according to which the average gradient of the velocity profile has a well-defined limit as the viscosity $\nu$ tends to zero [7], [9], [19]. This is the vanishing-viscosity principle. We thus expand the functions $A(R e), \alpha(R e)$ in powers of $\varepsilon$ and keep the first two terms; the result is

$$
\Phi=\left(A_{0}+A_{1} \varepsilon\right) \eta^{\alpha_{0}+\alpha, \varepsilon}
$$

in the range of $R e$ we shall be considering. Substitution of (4.9) into (4.7) yields

$$
\partial_{\ln \eta} \phi=\left(A_{0}+A_{1} \varepsilon\right) \eta^{\alpha_{0}+\alpha_{1} \varepsilon}=\left(A_{0}+A_{1} \varepsilon\right) e^{\left(\alpha_{0}+\alpha_{1} \varepsilon\right) \ln \eta} .
$$

The requirement that this quantity have a finite limit as $\nu$ tends to zero yields immediately $\alpha_{0}=0$ and shows that $\varepsilon$ must tend to zero as $R e$ tends to infinity like $\left(\frac{1}{\ln R e}\right)$ or faster. The assumption of incomplete similarity, experiment, and the vanishingviscosity principle show that the threshold value $\varepsilon=\frac{1}{\ln R e}$ is the proper choice. A substitution of this choice into equation (4.9) and an integration yield

$$
\phi=\frac{u}{u_{*}}=\left(C_{0} \ln R e+C_{1}\right) \eta^{\frac{\alpha_{1}}{\ln R e}},
$$

where the additional condition $\phi(0)=0$ has been used.

A useful property of the expression (4.11) is its asymptotic covariance [11], [27]. At large Re equation (4.11) should be invariant under a change in the definition of Reynolds number, which contains an arbitrary choice of length scale and velocity scale. A change in these choices multiplies $R e$ by a constant $Z$, and we expect formula (4.11) to remain valid, with the same $C_{0}, C_{1}, \alpha_{1}$, when $R e$ is replaced by $Z \cdot R e$. The obvious relation $\ln (Z R e)=\ln R e+\ln Z \sim \ln R e$ for large $R e$ ensures that equation (4.11) satisfies this requirement. 
According to this derivation, the coefficients $C_{0}, C_{1}, \alpha_{1}$ are universal constants, the same in all past and all future experiments of sufficiently high quality performed in pipe flows at large Reynolds numbers. In the paper [12] the proposed scaling law for smooth walls (4.11) was compared with what seemed to be the best available data, produced by Nikuradze [41] under the guidance of Prandtl at his institute in Göttingen. It is particularly important that these data are available in tabular form and not only as graphs. The comparison has yielded the coefficients $\alpha_{0}=0, \alpha_{1}=3 / 2$ $\left(C_{0}=\frac{1}{\sqrt{3}}, C_{1}=5 / 2\right)$, with an error of less than $1 \%$. For the details of the analysis of the experimental data, see [12]. Thus the final result is

$$
\phi=\left(\frac{1}{\sqrt{3}} \ln R e+\frac{5}{2}\right) \eta^{3 / 2 \ln R e},
$$

or, equivalently,

$$
\phi=\left(\frac{\sqrt{3}+5 \alpha}{\alpha}\right) \eta^{\alpha}, \quad \alpha=\frac{3}{2 \ln R e} .
$$

The proposed scaling law (4.11) produces a separate curve $\phi=\phi(\eta)$ in the $(\ln \eta, \phi)$ plane, one for each value of the Reynolds number $R e$, in contrast with the von Kármán-Prandtl law (1.7) which would produce a single curve for all values of Re.

We now wish to use the law (4.11) to understand what happens at larger Reynolds numbers and for a broader range of values of $\eta$ than were represented in the experiments reported by Nikuradze. If this extrapolation agrees with experiment, we can conclude that the law has predictive powers and provides a faithful representation of the intermediate region. We have already stated that the limit that must exist for descriptions of the mean gradient in turbulent flow is the vanishing-viscosity limit, and thus one should be able to extrapolate the law (4.11) to ever smaller viscosities $\nu$. This is different from simply increasing the Reynolds number, as $\nu$ affects $\eta$ and $\bar{u}$ as well as Re. Note that the decrease in the viscosity corresponds also to what is done in the experiments: if one stands at a fixed distance from the wall, in a specific pipe with a given pressure gradient, one is not free to vary $R e=\bar{u} d / \nu$ and $\eta=u_{*} y / \nu$ independently because the viscosity $\nu$ appears in both. If $\nu$ is decreased by the experimenter, the two quantities will increase in a self-consistent way, and $\bar{u}$ will vary as well. When one takes the limit of vanishing viscosity, one considers flows at ever larger $\eta$ at ever larger $R e$; the ratio $\frac{3 \ln \eta}{2 \ln R e}$ tends to $3 / 2$ because $\nu$ appears in the same way in both numerator and denominator. Consider the combination $3 \ln \eta / 2 \ln R e$. It can be represented in the form

$$
\frac{3 \ln \eta}{2 \ln R e}=\frac{3\left[\ln \frac{u_{*} d}{\nu}+\ln \frac{y}{d}\right]}{2\left[\ln \frac{u_{*} d}{\nu}+\ln \frac{\bar{u}}{u_{*}}\right]} .
$$

According to [3], at small $\nu$, i.e., large $R e, \bar{u} / u_{*} \sim \ln R e$, so that the term $\ln \left(\bar{u} / u_{*}\right)$ in the denominator of the right-hand side of (4.14) is asymptotically small, of the order of $\ln \ln R e$, and can be neglected at large $R e$. The crucial point is that, due to the small value of the viscosity $\nu$, the first term $\ln \left(u_{*} d / \nu\right)$ in both the numerator and denominator of (4.14) should be dominant, as long as the ratio $y / d$ remains bounded from below, for example, by a predetermined fraction. Thus, as long as one stays 
away from a suitable neighborhood of the wall, the ratio $3 \ln \eta / 2 \ln R e$ is close to $3 / 2$ ( $y$ is obviously bounded by $d / 2$ ). Therefore, the quantity

$$
1-\ln \eta / \ln R e
$$

can be considered as a small parameter, as long as $y>\Delta$, where $\Delta$ is an appropriate fraction of $d$. The quantity $\exp (3 \ln \eta / 2 \ln R e)$ is approximately equal to

$$
\begin{array}{r}
\exp \left[\frac{3}{2}-\frac{3}{2}\left(1-\frac{\ln \eta}{\ln R e}\right)\right] \approx \\
e^{3 / 2}\left[1-\frac{3}{2}\left(1-\frac{\ln \eta}{\ln R e}\right)\right] \\
=e^{3 / 2}\left[\frac{3}{2} \frac{\ln \eta}{\ln R e}-\frac{1}{2}\right]
\end{array}
$$

According to (4.7) we also have

$$
\eta \partial_{\eta} \phi=\partial_{\ln \eta} \phi=\left(\frac{\sqrt{3}}{2}+\frac{15}{4 \ln R e}\right) \exp \left(\frac{3 \ln \eta}{2 \ln R e}\right),
$$

and the approximation (4.15) can also be used in (4.16). Thus in the intermediate asymptotic range of distances $y: y>\Delta$, but at the same time $y$ slightly less than $d / 2$, we find, up to terms that vanish as the viscosity tends to zero,

$$
\phi=e^{3 / 2}\left(\frac{\sqrt{3}}{2}+\frac{15}{4 \ln R e}\right) \ln \eta-\frac{e^{3 / 2}}{2 \sqrt{3}} \ln R e-\frac{5}{4} e^{3 / 2},
$$

and

$$
\partial_{\ln \eta} \phi=\frac{\sqrt{3}}{2} e^{3 / 2} .
$$

We shall call equation (4.18) the asymptotic slope condition. It asserts that as $\nu \rightarrow 0$ the slope of the power law tends to a finite limit whose value, the limiting slope, is given by equation (4.18). In the von Kármán-Prandtl law an asymptotic slope condition is also assumed, with a limiting slope equal to $1 / \kappa$. Note that the limiting slope in equation (4.18), $\frac{\sqrt{3}}{2} e^{3 / 2}=1 / .2776$, is approximately $\sqrt{e} \sim 1.65$ larger than the generally accepted values for $\kappa^{-1}$. We emphasize that equation (4.18) contains no analog of the finite additive constant in a classical logarithmic law.

The analysis just given remains valid if $\Delta$, the lower bound on the range of $y$, tends to zero, provided it tends to zero with $\nu$ more slowly than $1 / \ln (1 / \nu)$.

Before explaining the difference in limiting slopes between the scaling law and the von Kármán-Prandtl law, we wish to point out the geometric significance of the vanishing viscosity limit in the scaling law. Note that the range of values of $\ln \eta=\ln u_{*} y / \nu$ that corresponds to a given portion of the pipe, say the one between the values $y=d / 40$ and $y=d / 2$, is constant, independent of $\nu$. On the other hand, the value of $\ln \eta$ that corresponds to the beginning of the range goes up as $\nu$ tends to zero. As the Reynolds number tends to infinity, the curves $\phi=\phi(\eta)$ given by the scaling law become flatter and their slope converges to $\frac{\sqrt{3}}{2} e^{3 / 2}$. Simultaneously, the window that corresponds to most of the pipe's cross-section moves to the right. For every finite value of $\nu$ we have a power law, but at vanishing viscosity the limit of the power law, within the range of values of $y$ that corresponds to the major part of 
the cross-section, can be represented asymptotically as a straight line. It is important for our analysis that the motion of the window and the flattening of the curves occur simultaneously as $\nu$ tends to zero. Thus, the asymptote of the power law occupies most of the pipe at a small enough viscosity; in the $(\ln \eta, \phi)$ plane it is located at infinity, because of the presence of $\nu$ in the definition of $\eta$.

It is easy to show that the family of curves $\phi=\phi(\eta)$ parametrized by $R e$ has an envelope, whose equation tends to

$$
\phi=\frac{1}{\kappa} \ln \eta+\frac{5}{2} e,
$$

with $\kappa=2 e / \sqrt{3}=.425 .$. , very close to the standard value of $\kappa$ found in the literature. The corresponding value of $\frac{1}{\kappa}$ is exactly $\sqrt{e}$ times smaller than the value on the righthand side of (4.18). It is natural to conjecture that the logarithmic law usually found in the literature corresponds to this envelope; indeed, if one plots points that correspond to many values of $R e$ on a single graph (as is natural if one happens to believe the von Kármán-Prandtl law (1.7)), then one is likely to become aware of the envelope. The visual impact of the envelope is magnified by the fact that the small $y$ part of the graph, where the envelope touches the individual curves, is stretched out in graphs such as Figure 2 by the effect of $\nu$ on the values of $\ln \eta$. Also, the measurements at very small values of $y$, where the difference between the power law and the envelope could be noticeable again, are missing because of experimental difficulties very near the wall. Thus, if our proposed scaling law is valid, the conventional logarithmic law is merely an illusion which substitutes the envelope of the family of curves for the curves themselves. The discrepancy of $\sqrt{e}$ between the slope of the curves and the slope of the envelope is the signature of the power law, and if observed in the data, it helps to decide whether the power law is valid. Note also that the family of curves (3.12) in the example at the end of section 3 also has an envelope, which is of little relevance to the structure of the individual members of the family for $y>0$. The situation is summarized in Figure 4, in which we show schematically the individual curves of the scaling law, their envelope, and the asymptotic slope.

Historically, the understanding of the flow in the intermediate region of wallbounded turbulence has been influenced by the well-known "overlap" argument due to Izakson, Millikan, and von Mises (IMM) (see, e.g., [22], [39]). This argument is also important in the history of matched asymptotic expansions. Its gist is that one can find general asymptotic forms for the velocity profile near the wall and for the velocity profile near the center of the pipe; these forms are assumed to match in the intermediate region, and the match reveals the intermediate asymptotics of that intermediate region. We have shown in an earlier publication [5] that the IMM argument in its original form starts from an assumption of complete similarity in the Reynolds number $R e$ and amounts to a demonstration that complete similarity in Re together with the asymptotic matching principle is sufficient to justify the von Kármán-Prandtl law. We now present a form of this argument that does not start from this sweeping and doubtful assumption.

Assume that from the wall outward one has a "wall law" of form

$$
\phi=u / u_{*}=f(\eta, R e),
$$

where $f$ is a dimensionless function; note that this form can be derived by our usual similarity argument. What is significant here is that we are not making use of the specific representation of $f$ that we have already derived. In the region adjacent to 


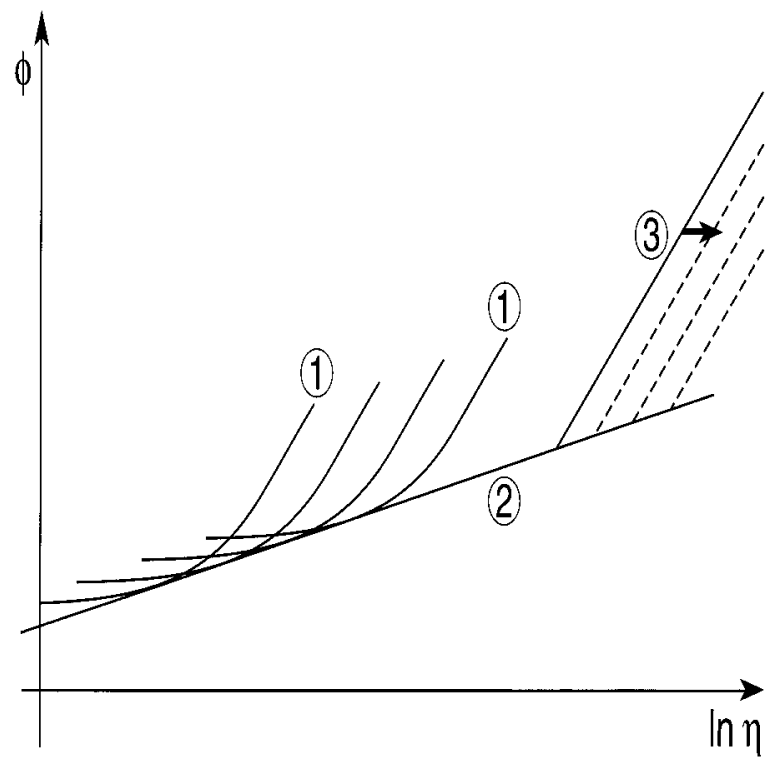

FIG. 4. Schematic of the power law curves, their envelope, and their asymptotic slope. 1. The individual curves of the scaling law. 2. The envelope of the family of scaling law curves (often mistaken for a logarithmic law of the wall). 3. The asymptotic slope of the scaling law curves.

the axis of the pipe the flow assumes a "defect law,"

$$
u_{C L}-u=u_{*} g(2 y / d, R e),
$$

where $u_{C L}$ is the average velocity at the centerline and $g$ is another dimensionless function. This form is chosen because it is natural to assume that far enough from the wall $\phi$ is no longer a function of a variable such as $\eta$ that emphasizes the influence of the wall. Asymptotic matching then demands that for some interval in $y$ the laws (4.20) and (4.21) overlap asymptotically, so that

$$
u_{C L}-u=u_{C L}-u_{*} f\left(u_{*} y / \nu, R e\right)=u_{*} g(2 y / d, R e),
$$

up to terms that are small when Re is large. After differentiation of (4.23) with respect to $y$ followed by multiplication by $y$ one obtains

$$
\eta \partial_{\eta} f(\eta, R e)=-\xi \partial_{\xi} g(\xi, R e)=G(R e),
$$

where $\eta=u_{*} y / \nu, \xi=2 y / d$, and $G(R e)$ is a function of $R e$ only. We now appeal to the vanishing-viscosity principle and find that $G(R e)$ must have a limit, say $G_{0}$, as $R e \rightarrow \infty$. Thus one half of equation (4.23) states that the function $\eta \partial_{\eta} f(\eta, R e)$ must have a constant limit when the viscosity tends to zero, and we already know (equation (4.18)) that our scaling law satisfies this condition. The other part of equation (4.23) serves to restrict the possible forms of the function $g$. Thus the IMM argument and our scaling law are perfectly compatible; indeed, one can derive the asymptotic form of the gradient of the scaling law from the IMM argument.

We wish to add two remarks. (i) Suppose one assumes complete similarity in Re in the foregoing argument; the function $G(R e)$ must then be a constant, and equation (4.24) then leads, after integration, to a logarithmic law, provided the additive constant can be defined. However, there are neither logical nor experimental reasons to 


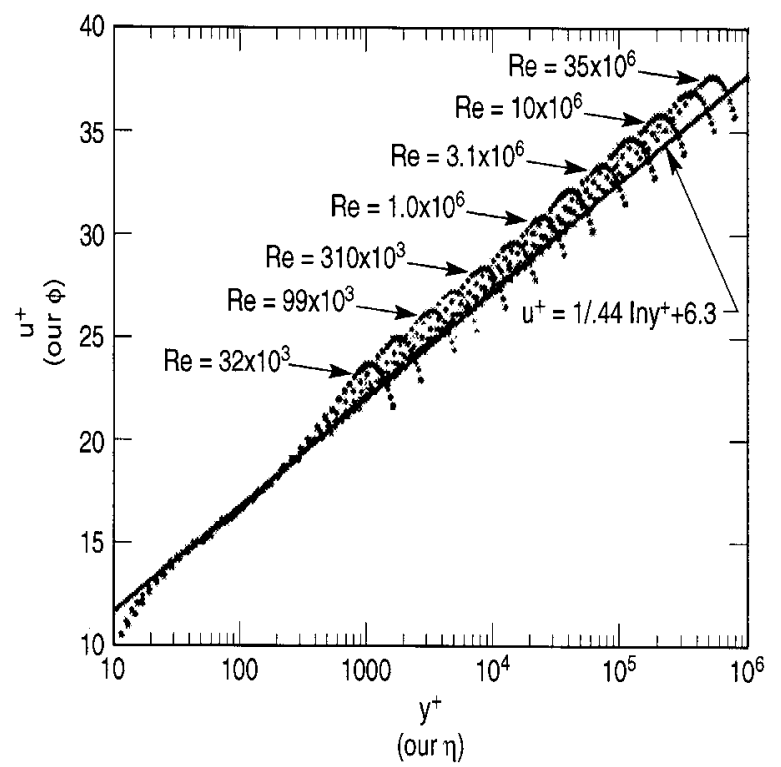

FIG. 5. The Princeton data [52] obtained in a high-pressure pipe confirm the splitting of the experimental data according to their Reynolds numbers and the rise of the curves above their envelope in the $(\ln \eta, \phi)$-plane. The solid line is the envelope; the curves turn at the center of the pipe. The splitting and form of the curves agree with the scaling law and are incompatible with the von Kármán-Pradtl universal logarithmic law. (Reproduced with permission from [51].)

make this assumption. (ii) The overlap argument suggests no value for the limiting constant $G_{0}$; if one decides to obtain this constant from the envelope of the family of velocity profiles rather than from the profiles themselves, one obtains the wrong value.

Finally, note that our description of the intermediate region in pipe flow is singular near the point $y=0$ as well as for $\nu=0$; the effective boundary condition is imposed on the intermediate region just outside the wall sublayer. Those are the features of our problem that have been built into the model problem at the end of the previous section.

5. The experimental data. Detailed comparisons of the power law and the von Kármán-Prandtl laws with experimental data are available in [8], [9], [12]. We shall be content here to show some experimental curves and corresponding profiles from our scaling law (4.7).

Figure 5 exhibits a series of mean velocity profiles in the $(\ln \eta, \phi)$ plane, as obtained in the Princeton experiments of Zagarola et al. [51], [52]. The curves turn down at the center of the pipe; the solid line is the conventional von Kármán-Prandtl law with the constants obtained in [51],[52], which differ from the conventional ones. Note that (i) there is a separate curve for each Reynolds number, in agreement with the power law (1.6) but not with the universal logarithmic law (1.7); (ii) the fact that the curves for various values of $R e$ were drawn on a single graph brings out the envelope of the family of curves; the range of values of $\ln \eta$ at which the envelope appears to be close to the individual graphs is exaggerated by the properties of $\ln \eta$ discussed above; (iii) each curve has a nearly straight upper part that forms a well-defined angle with the envelope; the ratio of the slopes is never less than 1.5. The curves appear staggered as a result of the use of $\ln \eta$ as ordinate. 


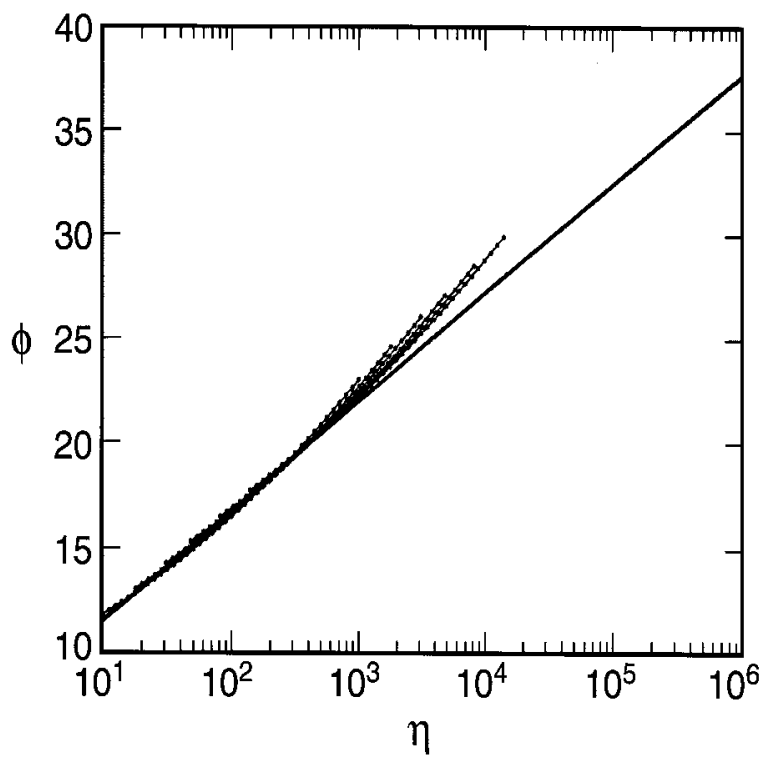

FIG. 6. The graphs of the individual power law curves for $R e \leq 10^{6}$ agree well with the experimental data.

These properties are properties of our proposed scaling law. Our claim is that the slope in the asymptotic slope condition is the slope to which the upper parts of the individual curves converge, while the usual slope in the von Kármán-Prandtl law is the slope of the envelope of the family of curves (see Figure 3). The same situation holds for the older data of Figure 2, where of course the upper parts of the profiles are less distinct because some of the Reynolds numbers are lower and thus the data points are closer to the envelope. Note also that some of these data are related to flows in boundary layers; note further that as the Reynolds number increases, the slope of the asymptotic slope condition approximates the slope of the individual curves over an increasing part of the pipe, as is logically necessary, while the illusory logarithmic law based on the envelope of the individual graphs approximates the real profiles over a decreasing part of the pipe. Similar data are available also for boundary layers (see, in particular, [28], [40]). These data are discussed in detail elsewhere [10].

These experimental graphs can be compared with the curves in Figures 6 and 7, which are derived from our scaling law (4.7). In Figure 6 we show only the first six profiles which agree very well with the experimental profiles. In Figure 7 we exhibit the profiles which correspond to all the curves in Figure 5. For the higher values of $R e$ there is some quantitative discrepancy between the theoretical and experimental profiles, which we have traced to roughness in the walls of the experimental set-up. At these high Reynolds numbers the thickness of the sublayer (region (1) in Figure 1 ) is a fraction of a micron; the small imperfections in the wall protrude from the sublayer and modify the flow. The importance of roughness is explained, e.g., in [39]; the blunting of the rise in $u$ as a result of roughness is shown in [16, p. 134]. In [8], [9] we showed how to allow for the roughness in the comparison of experimental and theoretical data and that once this allowance is made all the experimental curves agree with the scaling law quantitatively as well as qualitatively. In [9] it is also shown how the replacement of the von Kármán-Prandtl law by the scaling law brings quantities of interest to engineers (for example, the friction coefficient) into agreement with the experimental data. 


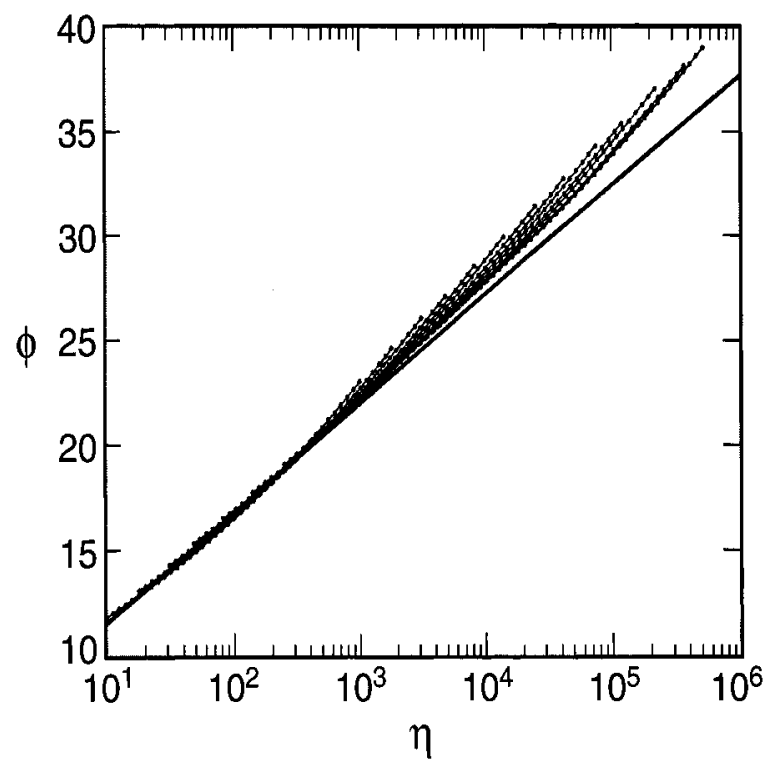

FIG. 7. The graphs of the individual power law curves for the same values of Re as in Figure 5. The experimental curves rise slightly more slowly than the theoretical curves because of roughness in the pipe's walls.

These figures make an additional important point: the dependence of the profiles on $\ln R e$ means that one needs a very high Reynolds number to see clearly the vanishing-viscosity limit of a turbulent flow. In general, it is dangerous to assume that available experimental data, and a fortiori numerical data, have reached the asymptotic regime in any manifestation of turbulence.

6. Local structure in turbulence. The analogy between the inertial range in the local structure of developed turbulence and the intermediate range in turbulent shear flow near a wall has been noted long ago (see, e.g., [17], [50]), and we appeal to it to motivate the extension of the scaling analysis above to the case of local structure, where the experimental data are much poorer. In the problem of local structure the quantities of interest are the higher moments of the relative velocity field, in particular, the second-order tensor

$$
D_{i j}=\left\langle\left(\Delta_{\mathbf{r}}\right)_{i}\left(\Delta_{\mathbf{r}}\right)_{j}\right\rangle,
$$

where $\Delta_{\mathbf{r}}=\mathbf{u}(\mathbf{x}+\mathbf{r})-\mathbf{u}(\mathbf{x})$ is a velocity difference between $\mathbf{x}$ and $\mathbf{x}+\mathbf{r}$. In incompressible flow all the components of this tensor are determined if one knows $D_{L L}=\left\langle\left[u_{L}(\mathbf{x}+\mathbf{r})-u_{L}(\mathbf{x})\right]^{2}\right\rangle$, where $u_{L}$ is the velocity component along the vector $\mathbf{r}$.

To derive an expression for the function $D_{L L}$ we assume, following the ideas of Kolmogorov, that for $r=|\mathbf{r}|$ small, it depends on the following quantities: $\langle\varepsilon\rangle$, the mean rate of energy dissipation per unit volume, $r$, the distance between the points at which the velocity is measured, a length scale $\Lambda$, which can be picked for convenience as the Taylor scale $\Lambda_{T}$ deduced from the correlation function of the overall flow, and the kinematic viscosity $\nu$; thus,

$$
D_{L L}(r)=f\left(\langle\varepsilon\rangle, r, \Lambda_{T}, \nu\right),
$$


where the unknown function $f$ is identical for all developed turbulent flows. If $r$ is large, other variables may appear as a consequence of external forces or boundary conditions. The most interesting, and also the most important, argument in this list is the rate of energy dissipation $\varepsilon$.

We now introduce the Kolmogorov scale $\Lambda_{K}$, which marks the lower bound of the "inertial" range of scales in which the energy dissipation is negligible:

$$
\Lambda_{K}=\frac{\nu^{3 / 4}}{\langle\varepsilon\rangle^{1 / 4}} .
$$

Clearly, the velocity scale appropriate to the inertial range is

$$
u=\left(\langle\varepsilon\rangle \Lambda_{T}\right)^{1 / 3},
$$

and this yields a Reynolds number

$$
R e=\frac{\left(\langle\varepsilon\rangle \Lambda_{T}\right)^{1 / 3} \Lambda_{T}}{\nu}=\frac{\langle\varepsilon\rangle^{1 / 3} \Lambda_{T}^{4 / 3}}{\nu}=\left(\frac{\Lambda_{T}}{\Lambda_{K}}\right)^{4 / 3} .
$$

We now consider the inertial range of scales, intermediate between the scales on which the fluid is stirred and the scales where viscosity dissipates its energy; it is the analog of the intermediate region we considered in wall-bounded flow. In that range the general scaling law that corresponds to (1.11) is

$$
D_{L L}=(\langle\varepsilon\rangle r)^{\frac{2}{3}} \Phi\left(\frac{r}{\Lambda_{K}}, R e\right),
$$

where as before, the function $\Phi$ is a dimensionless function of its two dimensionless arguments, which have been chosen so that under the circumstances of interest here they are both large.

If one now subjects the expression (6.5) to an assumption of complete similarity in both its arguments one obtains the classical Kolmogorov 2/3 law [34]

$$
D_{L L}=A_{0}(\langle\varepsilon\rangle r)^{\frac{2}{3}},
$$

from which the well-known Kolmogorov-Obukhov " $5 / 3$ " spectrum [42] can be obtained via a Fourier transform. If, on the other hand, one makes an assumption of incomplete similarity in $r / \Lambda_{K}$, one obtains a law of the form

$$
D_{L L}=A_{0}(\langle\varepsilon\rangle)^{\frac{2}{3}} r^{(2 / 3)+\alpha} \Lambda_{K}^{-\alpha},
$$

where $\alpha$ is an unknown correction to the exponent; the assumption that $\alpha$ is a universal constant corresponds to the "corrected" Kolmogorov-Obukhov theory (see [26], [35], [38], [39], [43]). Note, however, that $\Lambda_{K}$ tends to zero as the viscosity tends to zero, and if $D_{L L}$ has a finite limit when the viscosity tends to zero, as one may conclude from the statistical theory, the assumption that $\alpha$ is independent of $R e$ becomes untenable.

The most interesting assumption, as in the case of wall-bounded flow, is the assumption of incomplete similarity in $r / \Lambda_{K}$ and no similarity in $R e$. (In a subsequent paper [7], we shall show that this assumption is generically applicable in turbulence.) The result is

$$
\frac{D_{L L}(r)}{(\langle\varepsilon\rangle r)^{2 / 3}}=C(R e)\left(\frac{r}{\Lambda_{K}}\right)^{\alpha(R e)}
$$


where $C, \alpha$ are functions of $R e$ only. As before, we expand $C$ and $\alpha$ in powers of $\frac{1}{\ln R e}$ and keep the two leading terms; this yields

$$
D_{L L}=(\langle\varepsilon\rangle r)^{2 / 3}\left(C_{0}+\frac{C_{1}}{\ln R e}\right)\left(\frac{r}{\Lambda_{K}}\right)^{\alpha_{1} / \ln R e} .
$$

( $\alpha_{0}$ has been set equal to zero as the result of the requirement that $D_{L L}$ have a finite limit as $\nu \rightarrow 0$.) In the present problem, the molecular viscosity $\nu$ appears only in the variable $R e$, so that the limit of vanishing viscosity and the limit of infinite $R e$ coincide.

In real measurements for finite but accessibly large $R e, \alpha_{1} / \ln R e$ is small in comparison with 2/3, and the deviation in the power of $r$ in (6.9) could be unnoticeable. On the other hand, the variations in the "Kolmogorov constant" have been repeatedly noticed (see [45], [47]). Complete similarity is possible only if $A_{0} \neq 0$. If $A_{0} \neq 0$ one has a well-defined turbulent state with a $2 / 3$ law in the limit of vanishing viscosity, and finite $R e$ effects can presumably be obtained by expansion about that limiting state. In the limit of vanishing viscosity, there are no corrections to the "K-41" scaling if equation (6.10) holds; this conclusion was reached in [18] by the statistical mechanics argument summarized in section 2 above. We shall have more to say about the relation between the "K-41" scaling and the limit of infinite Reynolds number in the next section.

Kolmogorov [34] proposed similarity relations also for the higher order structure functions:

$$
D_{L L \ldots L}(r)=\left\langle\left[u_{L}(\mathbf{x}+\mathbf{r})-u_{L}(\mathbf{x})\right]^{p}\right\rangle,
$$

where $L L \ldots L$ denotes $L$ repeated $p$ times; the scaling gives $D_{L L \ldots L}=C_{p}(\langle\varepsilon\rangle r)^{p / 3}$. Experiments, mainly by Benzi et al. (see [13]), show some self-similarity in these higher-order functions, obviously incomplete, so that $D_{L L \ldots L}$ is proportional to $r^{\zeta_{p}}$, with exponents $\zeta_{p}$ always smaller then $p / 3$ for $p \geq 3$, so that $\zeta_{4}=1.28$ instead of 1.33, $\zeta_{5}=1.53$ instead of $1.67, \zeta_{6}=1.77$ instead of $2.00, \zeta_{7}=2.01$ instead of 2.33, and $\zeta_{8}=2.23$ instead of 2.67. It is tempting to try for an explanation of the same kind as for $p=2$ :

$$
D_{L L \ldots L}=\left(C_{p}^{0}+\frac{C_{p}^{1}}{\ln R e}\right)\left(\langle\varepsilon r\rangle^{p / 3}\left(r / \Lambda_{K}\right)^{\alpha_{p} / l n R e}\right) .
$$

In other words, it is assumed that at $R e=\infty$ the classic "K-41" theory would be valid, but the experiments were performed at Reynolds numbers too small to reveal the approach to complete similarity. If this explanation were correct, the coefficients $\alpha_{p}$ would be negative starting with $p=4$, where there would be a reversal in the effect of the Kolmogorov scale (or whatever scale was used to scale the first argument in $\Phi$ ).

As is well known, for $p=3$ the Kolmogorov scaling is valid with no corrections. For $p>3$, however, one must proceed with caution. We would like, however, to present a simple argument that casts doubt on the good behavior of the structure functions for integers $p>3$ in the vanishing-viscosity limit. Indeed, as $R e \rightarrow \infty$, the "active" regions of the flow shrink while energy is conserved. If $V_{0}$ is the fraction of the volume that corresponds to a unit mass of fluid where the kinetic energy $\approx u^{2}$ is large, then $u \approx \frac{1}{\sqrt{V_{0}}}$; one can easily see that fourth moments such as $\left\langle u^{4}\right\rangle$ diverge as $V_{0} \rightarrow 0$. This makes it likely that fourth-order structure functions also blow up (no conclusion 
can be drawn for $p=3$ because for odd powers $p$ cancellations can occur and the integrals can remain finite). Note that $p=3$ is the power where the sign of the power of $\frac{r}{\Lambda_{K}}$ in an expansion in powers of $\frac{1}{\ln R e}$ would change. The experimental results of Benzi et al. can be understood when one notices how slowly the vanishing-viscosity limit is approached.

A further comment relates what we have just discussed to the statistical theory of section 2: it may well be that the higher moments of $u$ require a longer time to relax to their equilibrium values than is available in a turbulent system; in this case the near-equilibrium theory does not apply to them and one cannot expect a valid smallviscosity limit. Indeed, according to Benzi's data [8], the time scale characteristic of moments of order $p$ increases with $p$ for $p>3$.

Finally, the discussion in the present section allows us to explain why, in the statistical analysis of section 2, one can assume that the small scales of turbulence have enough time to settle to an equilibrium, at least as far as an analysis of the lower order structure functions is concerned. At large $R e$, one can conclude from equation (6.10) that the characteristic velocity of an "eddy" of size $r$ is proportional to $r^{1 / 3}$; the characteristic time (length/velocity) is thus proportional to $r^{2 / 3}$ and tends to zero for small enough scales.

7. Intermittency. We have shown that when the viscosity is small the von Kármán-Prandtl universal logarithmic law for the intermediate region of wall-bounded shear flow must be replaced by a power law, of which we have offered a specific form that agrees with the data. It is interesting to consider what physical mechanisms produce this power law. We shall argue that the scaling law (4.6), (4.7) arises because the vorticity in the pipe is intermittent; i.e., most of the vorticity is concentrated on a small set. This intermittency, associated with the vorticity bursting process, is well documented in the experimental and numerical literature [14], [29], [33], [48], [49]. For historical reasons, intermittency is usually defined by the statement that most of the dissipation takes places in a small fraction of the available volume, and it is not obvious that the two definitions are equivalent. Ours is more convenient here. Intermittency can be viewed as a measure of the degree of turbulence in the flowthe more concentrated the vorticity is, the larger are the fluctuations whose presence defines fully developed turbulence.

A natural measure of the length scale of the cross-section of the transverse vortical structures near the wall, responsible for the vertical variation in the velocity $u$, is $\ell=\left(\partial_{y} u / u_{*}\right)^{-1}$. The scaling law (4.8) gives

$$
\ell=\frac{2}{\sqrt{3}+5 \alpha} y^{1-\alpha}\left(\nu / u_{*}\right)^{\alpha}, \quad \alpha=\frac{3}{2 \ln R e} .
$$

Note that $\ell$ is proportional to $y^{1-\alpha}$ rather than to $y$, showing that the transverse vortical structures are not space filling if (4.7) holds. One can define an essential support of the vorticity (see [19]) as the region where the absolute value of the vorticity exceeds some predetermined threshold; according to (7.1), the intersection of that essential support with a vertical line has fractal dimension $1-\alpha$. If the essential support is statistically invariant under translations parallel to the wall, the essential support itself has dimension $3-\alpha$. This conclusion agrees well with the data reported in [14], where the more powerful streamwise vortices are indeed not space filling. One could even hypothesize that, as the streamwise vortices meander, the transverse vortices that produce $u$ can be identified at least in part with transverse components of vortices that are mostly streamwise. 
An interpretation of these observations is suggested by the discussion in [49]. The process that occurs in a wall layer is a transfer of momentum (or impulse) from the outer regions to the wall, or, equivalently, a transfer of impulse of opposite polarity from the wall to the interior. This transfer is intermittent, concentrated in localized bursts of vorticity which create a vorticity scale different from $y$, consistent with the power law (4.7).

As the viscosity tends to zero, several things happen simultaneously: the slope of the scaling law approaches the asymptotic slope, the scale of the transverse vortices become proportional to $y$, the turbulence becomes highly intermittent, and one expects the vorticity to be concentrated on a small fraction of the available volume. One obtains a constant slope and a zero exponent $\alpha$ when the viscosity tends to zero and the turbulence is extremely intermittent, in the sense that most of the vorticity is concentrated in a very small volume. A nonzero viscosity, or equivalently, a finite Reynolds number, creates a correction to this ideal intermittency and produces a viscous correction to the asymptotic slope.

The explanation of this phenomenon, which runs counter to the usual analyses of the effect of intermittency, is straightforward: in the limit of vanishing viscosity one obtains a tangle of fractal vortex lines to which one can associate the scale of the volume that contains them. In the present case, this scale is $y$, and then the slope of the profile becomes constant. When the viscosity is finite, it exercises an ordering effect on the vortex lines (see [23], [24], [25]), creates a viscous scale for the organized vortices, and alters the scale of the transverse vortices. Thus the introduction of a small, finite viscosity into the problem creates a correction to the state of perfect intermittency and changes the asymptotic self-similar state. A small viscosity thus reveals the intermittency of the flow.

The asymptotic constant slope in the vanishing viscosity limit has a broader significance. The von Kármán-Prandtl law, which also has a constant slope, was derived on the basis of an assumption of complete similarity; i.e., it can be derived by standard dimensional analysis. Self-similar laws derived by dimensional analysis usually correspond to mean-field theories, i.e., statistical theories in which the effect of fluctuations on mean quantities has been neglected. The scaling law (4.7) has been derived on the basis of an assumption of incomplete similarity, the kind of assumption that leads to anomalous exponents and can describe theories in which fluctuations play a significant role. We have found that the vanishing viscosity limit of a theory with intermittency also leads to an asymptotic constant slope, just as the von KármánPrandtl law does, but it is not the slope usually identified with the von KármánPrandtl law, which is in fact the slope of the envelope of the scaling law curves. It is important to note that a specific qualitative conclusion (here, the existence of an asymptotic slope) can be derived from an incorrect similarity assumption and yet survive in a correct theory, albeit with a different interpretation and a different quantitative behavior. The example at the end of section 3 provides a simple example of this phenomenon, where the property in question is the constancy of the function $u(y, \delta)$. A similar phenomenon appears in the case of local structure.

Indeed, with the help of the analogy between wall-bounded turbulence and local structure, the discussion just given explains why there is no intermittency correction to the K-41 theory as $R e \rightarrow \infty$. When the limit is approached, there is no length scale attached to the vortices in the flow and no correction to the $2 / 3$ exponent. A small viscosity orders the flow and thus creates a new length scale and a correction. This argument gathers support from the analysis in [19], where a $2 / 3$ exponent was derived in a statistical theory where the vorticity was highly intermittent and the viscosity was absent. 
Further, the analysis explains why the Kolmogorov-Obukhov scaling succeeds for the second- and third-order functions but fails for the higher order functions. The Kolmogorov-Obukhov scaling, viewed as the limit as $R e \rightarrow \infty$ of the expressions one gets from an assumption of incomplete similarity, already takes into account the intermittency of the flow. This very intermittency is what makes the structure functions of high enough order diverge [7]. Thus, while we have recovered the form of the Kolmogorov-Obukhov scaling of the second- and third-order structure functions and thus of the energy spectrum, we are proposing a very different model for the underlying flow.

8. Conclusions. The following conclusions have been reached above. (i) A careful consideration of applicable similarity theory, in particular, the inclusion of functional forms suggested by incomplete similarity, broadens the range of possible scaling laws for the intermediate range of wall-bounded turbulence. A specific power law suggested by incomplete similarity is supported by the experimental data and is explained dynamically by the intermittency of the flow in the wall region when the viscosity is sufficiently small. This conclusion is significant in the numerical modeling of turbulence, where a wall law is often used as a boundary condition (see, e.g., [15]).

(ii) The analysis has been extended to the case of local structure in turbulence, where it leads to the conclusion that the corrections to the "K-41" theory are Reynolds-number-dependent and vanish in the limit $R e \rightarrow \infty$. The new interpretation of the "K-41" theory, as a limit of the scaling law derived from an assumption of incomplete similarity rather than as a result of complete similarity, makes it possible to view the resulting structure functions as corresponding to a highly intermittent flow field. In particular, there are no intermittency corrections to the Kolmogorov-Obukhov " $5 / 3$ " spectral exponent. The new interpretation of the Kolmogorov-Obukhov scaling is compatible with the breakdown of the scaling for high-order structure functions; it is supported by experimental evidence and is in full agreement with conclusions reached on the basis of the near-equilibrium statistical theory of vortex motion.

(iii) In the language of statistical physics, we have shown that exponents derived from the Kolmogorov-Obukhov scaling are not mean-field exponents, as is often claimed, but are the correct critical exponents for turbulence. More generally, the present work suggest that a workable statistical theory of turbulence is more likely to arise out of small-viscosity asymptotics than out of renormalized expansions in powers of a very large Reynolds number.

Acknowledgment. The authors would like to thank the following persons for helpful discussions and comments and/or for permission to use their data: Prof. N. Goldenfeld, Prof. O. H. Hald, Mr. M. Hites, Prof. F. Hussain, Prof. H. Nagib, Prof. C. Wark, and Dr. M. Zagarola.

\section{REFERENCES}

[1] G. I. Barenblatt, Similarity, Self-Similarity and Intermediate Asymptotics, Consultants Bureau, NY, 1979; Scaling, Self-Similarity, and Intermediate Asymptotics, Cambridge University Press, Cambridge, UK, 1996.

[2] G. I. BAREnblatt, On the scaling laws (incomplete self-similarity with respect to Reynolds number) in the developed turbulent flow in pipes, C. R. Acad. Sci. Paris, Ser. II, 313 (1991), pp. 307-312.

[3] G. I. Barenblatt, Scaling laws for fully developed turbulent shear flows, part 1: Basic hypotheses and analysis, J. Fluid Mech., 248 (1993), pp. 513-520.

[4] G. I. Barenblatt AND A. J. ChORIn, Small viscosity asymptotics for the inertial range of local structure and for the wall region of wall-bounded turbulence, Proc. Nat. Acad. Sci. USA, 93 (1996), pp. 6749-6752. 
[5] G. I. Barenblatt And A. J. Chorin, Scaling laws and vanishing viscosity limits for wallbounded shear flows and for local structure in developed turbulence, Comm. Pure Appl. Math., 50 (1997), pp. 381-398.

[6] G. I. BAREnblatt And A. J. ChORIn, Scaling laws and vanishing viscosity limits in turbulence theory, Proc. Sympos. Appl. Math., 54 (1997), pp. 1-25.

[7] G. I. Barenblatt And A. J. Chorin, A near-equilibrium statistical theory of turbulence with applications, 1997, in preparation.

[8] G. I. Barenblatt, A. J. Chorin, and V. M. Prostokishin, Scaling laws in turbulent pipe flow: Discussion of experimental data, Proc. Nat. Acad. Sci. USA, 94 (1997), pp. 773-776.

[9] G. I. Barenblatt, A. J. Chorin, And V. M. Prostokishin, Scaling laws for fully developed turbulent flow in pipes, Appl. Mech. Rev., 50 (1997), pp. 413-429.

[10] G. I. Barenblatt, A. J. Chorin, O. H. Hald, and V. M. Prostokishin, Structure of the zero-pressure-gradient turbulent boundary layer, Proc. Nat. Acad. Sci. USA, 94 (1997), pp. $7817-7819$.

[11] G. I. Barenblatt And N. Goldenfeld, Does fully developed turbulence exist? Reynolds number dependence vs. asymptotic covariance, Phys. Fluids A, 1995, pp. 3078-3082.

[12] G. I. Barenblatt And V. M. Prostokishin, Scaling laws for fully developed shear flows, part 2: Processing of experimental data, J. Fluid Mech., 248 (1993), pp. 521-529.

[13] R. Benzi, C. Ciliberto, C. Baudet, and G. Ruiz Chavarria, On the scaling of three dimensional homogeneous and isotropic turbulence, Phys. D, 80 (1995), pp. 385-398.

[14] P. Bernard, J. Thomas, And R. Handler, Vortex dynamics and the production of Reynolds stress, J. Fluid Mech., 253 (1993), pp. 385-419.

[15] H. B. Callen and T. A. Welton, Irreversibility and generalized noise, Phys. Rev., 83 (1951), pp. $34-40$

[16] T. Cebeci And A. M. O. Smith, Analysis of Turbulent Boundary Layers, Academic Press, New York, 1974

[17] A. J. Chorin, Theories of turbulence, in Berkeley Turbulence Seminar, P. Bernard and T. Ratiu, eds., Springer-Verlag, New York, 1977.

[18] A. J. Chorin, Equilibrium statistics of a vortex filament with applications, Comm. Math. Phys., 141 (1991), pp. 619-631.

[19] A. J. Chorin, Vorticity and Turbulence, Springer-Verlag, New York, 1994.

[20] A. J. Chorin, Turbulence as a near-equilibrium process, in Lectures in Appl. Math. 31, AMS, Providence, RI, 1996, pp. 235-248.

[21] A. J. ChoRin, Turbulence cascades across equilibrium spectra, Phys. Rev. E, 54 (1996) pp. 2616-2619.

[22] D. Coles, The law of the wall in the turbulent boundary layer, J. Fluid Mech., 1 (1956), pp. 191-226.

[23] P. Constantin, Navier-Stokes equations and area of interfaces, Comm. Math. Phys., 129 (1990), pp. 241-266.

[24] P. Constantin, Geometric and analytic studies in turbulence, in Trends and Perspectives in Applied Mathematics, L. Sirovich, ed., Springer-Verlag, New York, 1991, pp. 21-54.

[25] P. Constantin, Geometric statistics in turbulence, SIAM Rev., 36 (1994), pp. 73-98.

[26] U. Frisch, Turbulence: The Legacy of A. N. Kolmogorov, Cambridge University Press, Cambridge, UK, 1995.

[27] N. Goldenfeld, Lectures on Phase Transitions and the Renormalization Group, AddisonWesley, Reading, MA, 1992.

[28] M. Hites, Scaling of the High-Reynolds Number Turbulent Boundary Layer, Ph.D. thesis, Illinois Institute of Technology, 1997.

[29] P. Kailasnath, Reynolds Number Effects and the Momentum Flux in Turbulent Boundary Layers, Ph.D. thesis, Yale University, New Haven, CT, 1993.

[30] Th. von Kármán, Mechanische Aehnlichkeit und Turbulenz, Nach. Ges. Wiss. Goettingen Math-Phys. Klasse, 1932, pp. 58-76.

[31] T. KATO, Remarks on the zero viscosity limit for nonstationary flows with boundary, in Seminar on Partial Differential Equations, S.S. Chern, ed., Springer-Verlag, New York, 1984, pp. 8598.

[32] R. Klein And A. Majda, Self-stretching of a perturbed vortex filament, Phys. D 49 (1991), pp. 323-342.

[33] S. J. Kline, W. C. Reynolds, F. A. Schraub, and P. W. Rundstadler, The structure of turbulent boundary layers, J. Fluid Mech., 30 (1967), pp. 741-774.

[34] A. N. Kolmogorov, Local structure of turbulence in incompressible fluid at a very high Reynolds number, Dokl. Acad. Sci. USSR, 30 (1941), pp. 299-302. 
[35] A. N. Kolmogorov, A refinement of previous hypotheses concerning the local structure of turbulence in a viscous incompressible fluid at a high Reynolds number, J. Fluid Mech., 13 (1962), pp. 82-85.

[36] L. D. Landau And E. M. Lifshitz, Fluid Mechanics, Pergamon, New York, 1959.

[37] M. Lesieur, Turbulence in Fluids, Kluwer Academic, Dordrecht, the Netherlands, 1990.

[38] W. D. МсCомв, Physical Theories of Turbulence, Oxford University Press, Oxford, UK, 1991.

[39] A. S. Monin And A. M. Yaglom, Statistical Fluid Mechanics, Vol. 1, MIT Press, Boston, MA, 1971.

[40] H. NAGib And M. Hites, High Reynolds Number Boundary Layer Measurements in the NDF, AIAA paper 95-0786, Reno, NV, 1995.

[41] J. NikURAdZE, Gesetzmaessigkeiten der turbulenten Stroemung in glatten Rohren, VDI Forschungheft, 356, 1932.

[42] A. M. OвukHov, Spectral energy distribution in turbulent flow, Dokl. Akad. Nauk USSR, 1 (1941), pp. 22-24

[43] A. M. OвukHov, Some specific features of atmospheric turbulence, J. Fluid Mech., 13 (1962), pp. $77-81$.

[44] L. Prandth, Zur turbulenten Stroemung in Rohren und laengs Platten, Ergeb. Aerodyn. Versuch., Ser. 4, Goettingen, Germany, 1932.

[45] A. Praskovsky and S. Oncley, Measurements of the Kolmogorov constant and intermittency exponents at very high Reynolds numbers, Phys. Fluids A, 6 (1994), pp. 2778-2784.

[46] H. Schlichting, Boundary Layer Theory, 2nd ed., McGraw-Hill, New York, 1968.

[47] K. R. Sreenivasan, On the universality of the Kolmogorov constant, Phys. Fluids A, 7 (1994), pp. $2778-2784$.

[48] K. R. Sreenivasan, A unified view of the origin and morphology of turbulent boundary layer structure, IUTAM Symp., Bangalore, H. W. Liepmann and R. Narasimha, eds., VDIVerlag, Berlin, 1987.

[49] K. R. SReEnivasan, The turbulent boundary layer, in Frontiers in Experimental Fluid Mechanics, M. Gad-el-Hak, ed., Springer-Verlag, New York, 1989, pp. 159-209.

[50] H. Tennekes and J. Lumley, A First Course in Turbulence, MIT Press, Cambridge, MA, 1990.

[51] M. V. Zagarola, A. J. Smits, S. A. Orszag, And V. Yakhot, Experiments in High Reynolds Number Turbulent Pipe Flow, AIAA paper 96-0654, Reno, NV, 1996.

[52] M. V. Zagarola, Mean Flow Scaling in Turbulent Pipe Flow, Ph.D. thesis, Princeton University, Princeton, NJ, 1996. 